\title{
INTERFEROMETRIC MEASUREMENTS OF ANNULUS-FILTERED POLARIZATIONS PATTERNS IN THE CMB
}

\author{
CHENG-JIUN MA \\ cjma@asiaa.sinica.edu.tw \\ TZIHONG CHIUEH * \\ chiuehth@phys.ntu.edu.tw \\ Physics Department, National Taiwan University, \\ No.1, Sec. 4, Roosevelt Rd., Taipei, Taiwan (106)
}

\begin{abstract}
Due to the finite-size beam, interferometry observations of the CMB polarization tend to measure a mixture of $E$ and $B$ modes arising from $u v$-space smearing. We propose an interferometry observation strategy that can disentangle the polarization $E$ and $B$ modes in a clean manner. This strategy employs a similar principle as that of the singledish observation proposed earlier, but requiring additional linear combinations of signals measured from different baselines. In this work, we consider a hexagonally packed interferometry array as an example to demonstrate the ultility and efficiency of the proposed method. The measurement from a unit cell of equilateral triangular baselines in interferometry measurement is found to be comparable to that from a single dish in single-dish measurements.
\end{abstract}

Keywords: cosmic microwave background radiation; polarization

\section{Introduction}

Recently, DASI ${ }^{6}$, WMAP ${ }^{5}$, and Boomerang ${ }^{8}$ have successfully detected the polarization of cosmic microwave background (CMB), and several future experiments (QUaD, POLARBeaR, SPTpol, CMBpol and PLANCK) propose to measure the $B$ mode polarization. The primordial $B$ mode polarization is believed to be caused by primordial gravitational waves and provides information of inflation energy scale ${ }^{11}$. On the other hand, the secondary $B$ mode polarization induced by gravitational lensing of CMB will give us another probe of large scale density distribution ${ }^{4}$. However, the weak intensity of $B$ mode polarization makes it difficult to be measured cleanly in the presence of $E$ mode. Contamination by $E$ mode arises from the limitation of practical observation, such as incomplete sky coverage, finite resolution, and the foreground effects; the way to solve $E-B$ separation problem for real-space maps is also discussed in Ref. 14, Ref. 12, Ref. 2, Ref. 7 and Ref. 1.

For interferometric measurements, the $E$ and $B$ modes of CMB polarization can be represented as the projections of the Fourier components of the Stokes

\footnotetext{
*Adjunt Research Fellow, Institute of Astronomy and Astrophysics, Academia Sinica, Taipei,
} Taiwan 
parameters $^{13}, \mathrm{Q}$ and $\mathrm{U}$, onto different directions with respect to the baseline $\mathbf{k}$. Hence, interferometry which derives amplitude and phase in $\mathrm{k}$-space directly seems able to extract $E$ and $B$ modes straightforwardly. However, the finite dish size, especially in the case of a close-packed array, optimized for the sensitivity to CMB, makes the baseline $\mathbf{k}$, and thus the directional projections, ill-defined, thereby mixing up $E$ and $B$ modes in the measurements.

In this work, we show that clean separation of $E$ and $B$ modes can actually be achieved with interferometric measurements by scanning along a sky annulus. A similar method has been proposed for single-dish measurement in our previous work ${ }^{2}$. Different from the conventional interferometry observations of CMB polarization $^{13,9,10}$, which have afore-mentioned difficulties, our proposed strategy incorporates the advantage of real-space measurements that warrants separation of $E$ and $B$ modes in practice.

\section{Observation strategy and simulation}

The polarization field can be expressed in the form of second order derivatives of 2 scalar fields $f$ and $g$, from which the $E$ and $B$ tensor modes are generated respectively. For convenience, it is written in polar coordinates as

$$
\begin{aligned}
\mathbf{P}=Q+i U & =4 \frac{\partial^{2}}{\partial \bar{w}^{2}}(f+i g) \\
& \left.=e^{2 i \phi}\left[r \frac{\partial}{\partial r}\left(\frac{1}{r} \frac{\partial}{\partial r}\right)-\frac{1}{r^{2}} \frac{\partial^{2}}{\partial \phi^{2}}\right)+\frac{2 i}{r} \frac{\partial}{\partial \phi}\left(\frac{\partial}{\partial r}-\frac{1}{r}\right)\right](f+i g) .
\end{aligned}
$$

The map generated by interferometric array is constructed by the visibility (i.e., Fourier transformation) of the beam-weighted map $(\mathbf{P})$ integrated over the selected sky. In previous work ${ }^{2}$, we have suggested that the $E$ and $B$ modes can be cleanly separated in a real-space map if one adopts an annulus filter function. In practice, it employs scanning in a sky circle where the polarization axes also rotate synchronously. The radius $R_{q}$ of the scanning circle as well as the primary beam size $D$ are free parameters to be optimized according to the $l$-range of interest and the responsivity of measurements. Such an idea can be carried over to the interferometric measurement. For simplicity of the following analysis, we assume a Gaussian beam.

The visibility of interferometry of baseline $k$ with annulus filtering could be expressed as:

$$
\begin{aligned}
& \Delta T\left(k ; R_{q}, D\right)=e^{-i k R_{q} \cos \phi_{k 0}} \int_{\phi_{k^{\prime}}>0} e^{-\left(k^{2}+k^{\prime 2}\right) D^{2} / 2} \\
& \sum_{n=-\infty}^{\infty} I_{n}\left(k k^{\prime} D^{2}\right) J_{n+2}\left(k^{\prime} R_{q}\right) e^{i n\left(\pi / 2-\phi_{k 0}\right)}\left(k^{\prime}\right)^{2}\left(\operatorname{Re}\left[f_{\mathbf{k}^{\prime}}\right]+i \operatorname{Re}\left[g_{\mathbf{k}^{\prime}}\right]\right) \frac{d^{2} \mathbf{k}^{\prime}}{2 \pi^{2}}
\end{aligned}
$$

where $J_{\mathrm{s}}$ and $I_{\mathrm{s}}$ are (modified) Bessel functions. Following the convention of eqn (1), the real/imaginary part of the measurement corresponds to the Stokes $Q / U$ respectively. Obviously, the exponential term in eqn (2) mixes up $f$ and $g$, the 
sources of $E$ and $B$ modes, in the measured $Q$ and $U$. Nevertheless the mixing can be disentangled by linearly combining the data measured by any pair of baselines that satisfy the "separation condition", $\phi_{k b}=(2 n+1) \pi-\phi_{k a}$ ( $\phi_{k}$ is the phase angle of the baseline). The resulting visibility is

$$
\begin{aligned}
& \Delta T_{ \pm}\left(k ; R_{q}, D\right)=\frac{1}{\sqrt{2}}\left(e^{i k R_{q} \cos \phi_{k a}} \Delta T_{a}\left(k ; R_{q}, D\right) \pm e^{i k R_{q} \cos \phi_{k b}} \Delta T_{b}\left(k ; R_{q}, D\right)\right) \\
& =\sqrt{2} \int_{\phi_{k^{\prime}}>0} e^{-\left(k^{2}+k^{\prime 2}\right) D^{2} / 2} \\
& \quad \sum_{n=-\infty}^{\infty} I_{n}\left(k k^{\prime} D^{2}\right) J_{n+2}\left(k^{\prime} R_{q}\right) G_{ \pm}\left(\phi_{k a}\right) k^{\prime 2}\left(\operatorname{Re}\left[f_{\mathbf{k}^{\prime}}\right]+i \operatorname{Re}\left[g_{\mathbf{k}^{\prime}}\right]\right) \frac{d^{2} \mathbf{k}^{\prime}}{2 \pi^{2}}
\end{aligned}
$$

where $G_{+}\left(\phi_{k a}\right) \equiv \cos \left(n\left(\phi_{k a}+\pi / 2\right)\right)$ and $G_{-}\left(\phi_{k a}\right) \equiv i \sin \left(n\left(\phi_{k a}+\pi / 2\right)\right)$. In addition, the square variance of $\Delta T_{ \pm}\left(k ; R_{q}, D\right)$ can be calculated in terms of the conventional angular power spectrum $l(l+1) C_{l} / 2 \pi$.

Fortunately, the baselines of the most compact configuration, hexagonal packed, all satisfy the separation condition. These equilateral triangle baselines can be categorized into 2 configurations. The $\alpha$-configuration has baselines subtending from the radial direction with angles $\phi_{k 0}=0, \pi / 3,2 \pi / 3, \pi, 4 \pi / 3,5 \pi / 3$, and the $\beta$-configuration with angles $\phi_{k 0}=\pi / 6, \pi / 2,5 \pi / 6,3 \pi / 2,7 \pi / 6,11 \pi / 6$.

The squared variance of primordial CMB polarization in $\Lambda$ CDM cosmology is plotted against the annulus ring radius $R_{q}$ and beam size $D$ in Fig 1 . As a reference, the result of single dish measurement is also plotted with some beam sizes.
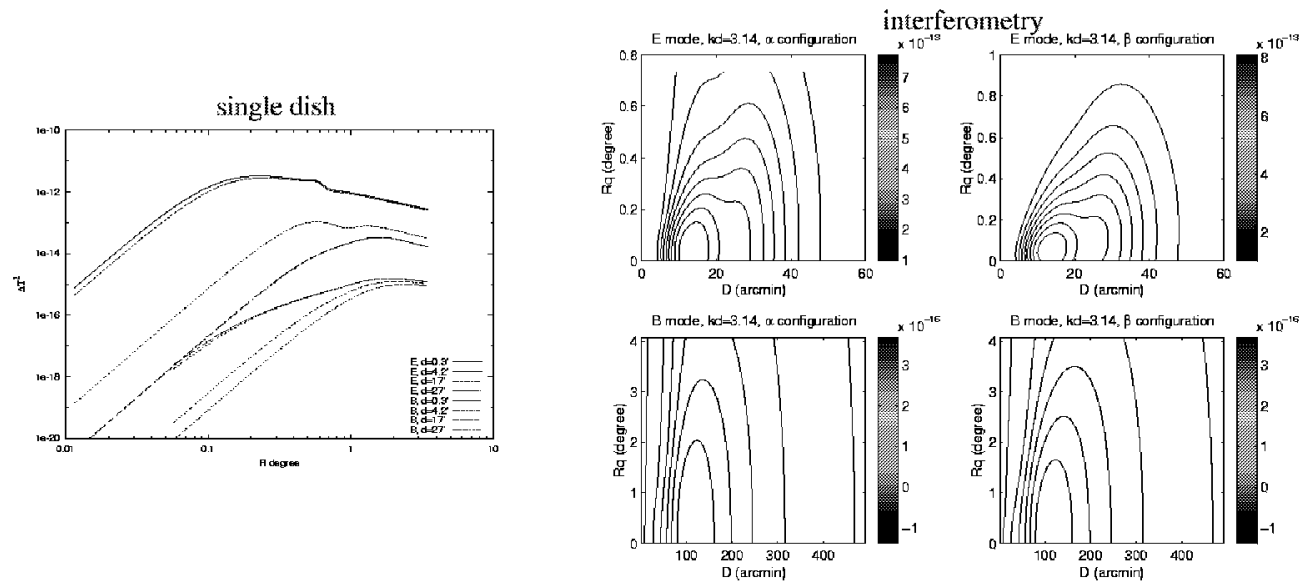

Fig. 1. On the left hand side, it is the surface brightness $\left(\Delta T_{r m s}^{2}\right.$ in $\left.\mathrm{K}\right)$ of E-mode and B-mode polarization of single dish measurement.Smaller beam size, i.e., larger dish, has better sensitivity. On the right hand side, the contour is the surface brightness $\left(\Delta T_{r m s}^{2}\right.$ in $\left.\mathrm{K}\right)$ of $\mathrm{E}$-mode and $\mathrm{B}$ mode polarization with $\alpha$-configuration and $\beta$-configuration of an interferometry. The optimized dish size is small, $8 \mathrm{~cm}$, in $90 \mathrm{GHz}$. 


\section{Discussion}

We demonstrate that interferometry measurements with the scanning annulus observing strategy can be clean in separating $E$ and $B$ modes and involving no statistical methods for $E-B$ separation employed conventionally ${ }^{12}$. Even pixelization of data can be bypassed here, in that we measure the continuously integrated surface brightness on a sky annulus. Because of the simplicity of this strategy, the quantity derived above can be used for an indication of $B$ mode detection.

Comparing the two figures, the responsivity to the primordial $B$ mode polarization for one single dish (the $k=0$ case) is 6 times higher than that for one baseline. When the dish number $N$ is large, the performance ratio between the $N$-single-dish measurements and the $N$-dish interferometry measurement of close packed configuration drops to two. An experiment targeting at the CMB $B$-mode can be conducted on a turning table that houses many $\sim 10 \mathrm{~cm}$ dishes. In such a setup, one can perform both interferometry measurement and (many) single-dish measurement at the same time. Though the small dish size is not optimal in sensitivity for single-dish measurement, it helps suppress $E$ modes much more than $B$ modes and serves to further avoid accidental $E$-mode leakage ${ }^{2}$. In view of recent advents in the integrated MMIC technology for CMB detectors ${ }^{3}$, such a CMB B-mode experiment is expected to be realizable in the foreseeable future.

\section{Acknowledgments}

This work is supported in part by the National Science Council of Taiwan under the grant NSC90-2112-M-002-026. The CMB power spectrum is obtained from CMBFAST.

\section{References}

1. E. F. Bunn, et al. Phys. Rec. D., 67, 3501, (2002)

2. T. Chiueh \& C-J. Ma, ApJ, 578:12-24, (2002)

3. T. Gaier, et al. NewAR, 47, 1167, (2003)

4. C. M. Hirata \& U. Seljak, Phys. Rev. D, 68, 083002, (2003)

5. Kogut et al., ApJS, 148, 161, (2003)

6. J. M. Kovac, et al. Nature, 420, 772, (2002)

7. A. Lewis, A. Challinor, \& N. Turok, Phys. Rev. D., 65, 3505, (2002)

8. T. Montroy et al, NewAR, 47, 11, 1057, (2003)

9. K.-W. Ng, Phys. Rev. D., 63, 123001, (2001)

10. C. G. Park, \& K.-W. Ng, arXiv:astro-ph/0305545

11. U. Seljak, \& M. Zaldarriaga, ApJ, 469, 437, (1996)

12. M. Tegmark, \& A. de Oliveira-Costa, Phys. Rec. D., 64, 063001, (2001)

13. M. White, J. E. Carlstrom, M. Dragovan, \& W. L. Holzapfel, ApJ, 514:12-24, (1999)

14. M. Zaldarriaga, Phys. Rev. D, 64, 103001, (2001) 